\title{
Electroneurographic correlates of the monosynaptic reflex: experimental studies and normative data
}

\author{
MICHELE ABBRUZZESE, SANDRO RATTO, GIOVANNI ABBRUZZESE, \\ EMILIO FAVALE
}

From the Department of Neurology, University of Genoa, Genoa, Italy

SUMMARY The neurographic concomitants of the monosynaptic reflex, evoked either by electrical stimulation of the tibial nerve at the popliteal fossa or by percussion of the Achilles tendon, have been recorded from the sciatic nerve in the lower and middle thigh. Neurographic recordings were characterised by two travelling waves $\left(P_{1}\right.$ and $\left.P_{2}\right)$, respectively increasing and decreasing in latency in the proximal direction, that showed the same chronological trend of the propagated action potentials concurrently recorded in the dorsal and ventral spinal roots at the lumbar level. At variance with $P_{2}$, the speed of propagation of the $P_{1}$ volley was stimulus-related, being faster on mechanical than on electrical stimulation, probably because in the latter case the latency of the fastest afferents is overestimated. The $P_{2}$ volley is subserved by $\alpha$-efferent fibres in either case as suggested, inter alia, by the strict parallelism between the $\mathrm{P}_{2}$ volley and the monosynaptic reflex under appropriate experimental conditions. Simultaneous recordings of spinal root and sciatic nerve action potentials allowed the direct assessment of afferent and efferent conduction vel ocities, both in the proximal (that is from the middle thigh to the spinal recording site and vice-versa) and in the distal (that is from the lower to the middle thigh recording site and vice versa) segments of the reflex arc. As expected, the speed of propagation of impulses was significantly higher in the proximal than in the distal segments, as well as in the afferent than im efferent limb of the monosynaptic pathway. The $P_{1}-P_{2}$ time interval was longer on mechanical than on electrical stimulation, probably due to the increased spinal delay of the $\mathrm{T}$ versus the $\mathrm{F}_{\mathrm{S}}$ reflex. The present study provides a reliable method for the direct assessment of $\alpha$-efferent as well as of Ia afferent group fibres conduction velocity, provided that in the latter case mechanical stimuli be used.

The evaluation of the neural concomitants of the monosynaptic reflex provides a unique opportunity to assess directly the conduction velocity of the motor nerve fibres as well as of group Ia afferent fibres provided that, in the latter case, mechanical stimuli be employed. Surprisingly, in the few systematic studies dedicated to the neurographic correlates of electrically ${ }^{1}$ or mechanically ${ }^{2}$ elicited monosynaptic reflexes in man, no centrifugal potentials in the peripheral nerve could be recorded. This failure, accounted for by the temporal dispersion of the efferent volley ${ }^{32}$ contrasts with the results of a recent investigation, which show that reproducible surface

Address for reprint requests: Michele Abbruzzese MD, Clinica Neurologica, Via De Toni 5, I 16132 Genova, Italy.

Received 30 July 1984.

Accepted 27 September 1984 recordings of the ventral spinal root discharge can be obtained when the stimulus intensity is adjusted to elicit a maximal $\mathrm{H}$ reflex, and also with some occasional observations demonstrating that a reflexly induced discharge in $\alpha$-efferent fibres can be recorded from the tibial nerve on either mechani$\mathrm{cal}^{4-6}$ or electrical ${ }^{6}$ stimulation at appropriate intensities.

The present study demonstrates that well defined neurographic recordings not only of the afferent but also of the efferent activity underlying the monosynaptic reflex can be obtained easily from cooperative normoreflexic subjects, the behaviour of such neurographic responses being consistent with that of the monosynaptic reflex under appropriate experimental conditions. Moreover it will be shown that both proximal and distal conduction velocities through the monosynaptic reflex pathway can be 
assessed directly by concurrent recordings of spinal root and peripheral nerve action potentials.

\section{Materials and methods}

Experiments were carried out in a quiet room on 32 fully informed healthy volunteers, aged $24-48$ years; some were examined on several occasions. During the experiment the subject lay prone with the head rotated towards the stimulated side and the leg under study supported on cushions. The hip was extended, the knee slightly flexed and the foot, positioned in about $10^{\circ}$ plantar flexion, fixed on a metal baseplate. The legs were gently warmed by an infrared lamp to ensure a skin temperature of $36-38^{\circ} \mathrm{C}$. The tibial nerve was stimulated using surface electrodes with the cathode $\left(2 \mathrm{~cm}^{2}\right)$ in the popliteal fossa and the anode $\left(20 \mathrm{~cm}^{2}\right)$ over the patella. If stimulus artefact presented a problem, bipolar stimulation was used. Square wave pulses were delivered by Disa $15 \mathrm{E} 25$ stimulator output (pulse duration $0.5 \mathrm{~ms}$ ) at a rate of $0.5 \mathrm{~Hz}$ except when the effects of increasing frequencies (from 0.5 to $5.0 \mathrm{~Hz}$ ) were studied. The stimulus intensity was adjusted to elicit a maximal $\mathrm{H}$ reflex, the whole range of intensities being used when the effects of increasing intensities were investigated. In no case was it necessary to use intensities higher than $30 \mathrm{~mA}$. The Achilles tendon was percussed manually using a reflex hammer with a built-in microswitch capable of triggering the sweep of the oscilloscope. The trigger delay was $0.2 \mathrm{~ms}$. Tendon taps at constant strength were delivered at a frequency of about one every $5 \mathrm{~s}$.

Responses evoked by either electrical or mechanical stimulation were recorded from lumbar spine and from peripheral nerve. Spinal recordings were performed using fine bare subcutaneous needles with an electrode impedance of 1-2 $\mathrm{kOhm}$, and an interelectrode distance of 5-7 cm. The caudal electrode was placed over the spinous process of $\mathrm{L} 4$ and connected to input terminal 1 of the amplifier. Alternatively, the responses were recorded using long insulated steel needle electrodes (Disa 13L63) placed within the spinal theca across the interspaces L3-4 and L4-5, and referenced to an uninsulated subcutaneous needle at the same vertebral level. Neural activity was also recorded from the sciatic nerve, at lower (LT) and middle (MT) thigh, using insulated steel needle electrodes placed close to the trunk of the nerve. In particular, two electrode arrays were employed: (a) the active electrode was placed near the nerve, the remote lead being at a transverse distance of $2 \mathrm{~cm}$; (b) both electrodes were placed along the nerve axis, the interelectrode distance being about $1 \mathrm{~cm}$. To guide its insertion, the active electrode was initially used for stimulation and its position adjusted until the threshold of the soleus muscle action potential was $0.5-1.0$ $\mathrm{mA}$. The electromyographic responses (that is $\mathrm{H}, \mathrm{M}$ and $\mathrm{T}$ ) were recorded using surface electrodes (Disa 13K60) placed $3 \mathrm{~cm}$ apart on the skin overlying the soleus muscle, the active lead being proximal.

The input from the lumbar and the thigh electrodes was fed into differential amplifiers (Disa 15C52) and simultaneously recorded on magnetic tape (Philips Analog 7). The frequency responses of the recording apparatus was $10-8.000 \mathrm{~Hz}(3 \mathrm{~dB})$. Analysis times of 20 or $50 \mathrm{~ms}$ were used and 8-16 responses to electrical stimulation or 64-
128 responses to mechanical stimulation were summated by means of an averager (Disa $15 \mathrm{G} 07,100 \mathrm{kHz}$ sampling rate) and displayed on X-Y plotter (Philips PM 8125). Number, shape and polarity were analysed visually. Latencies were measured at the first positive peak with on the plot or, with a cursor, on the average oscilloscope. The EMG responses were amplified, averaged and displayed by a Disa System 1500.

Vibration of the calf muscle on the stimulated side was undertaken by means of a cylindrical vibrator (Heiwa, Model TMT 18) (vibration frequency $100 \mathrm{~Hz}$, amplitude $2 \mathrm{~mm}$ ) applied with a constant pressure over the Achilles tendon.

The results were evaluated statistically by means of the Student $t$ test for paired data, the level of significance being conservatively established at $\mathrm{p}<0.005$.

\section{Results}

Neurographic correlates $\left(P_{1}\right.$ and $P_{2}$ volleys) of the $H$ reflex

At stimulus intensities yielding a maximal $\mathrm{H}$ reflex, well defined, reproducible responses could be recorded in all 32 subjects from the sciatic nerve, both in the lower (LT) and middle (MT) thigh. The neural recordings were characterised by two

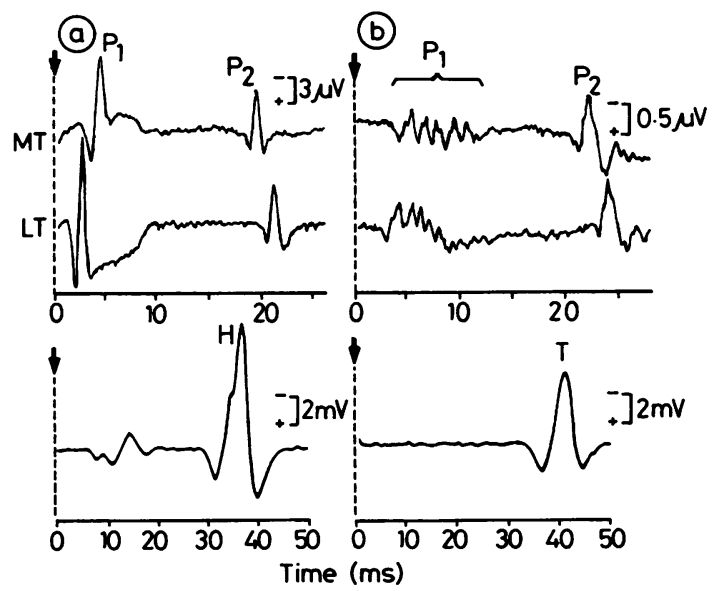

Fig 1 Electroneurographic and electromyographic monopolar recordings of the responses evoked (a) by electrical stimulation of the tibial nerve and (b) by percussion of the Achilles tendon. The electroneurographic responses, recorded from the sciatic nerve at the lower (LT) and the middle (MT) thigh, are characterised by two travelling waves $\left(P_{1}\right.$ and $\left.P_{2}\right)$, respectively increasing and decreasing in latency in the proximal direction. Amplitude, latency, shape and duration of both $P_{1}$ and $P_{2}$ are remarkably different according to the stimulus used. The time interval between $P_{1}$ and $P_{2}$ is longer on the mechanical than on the electrical stimulation, both at MT and LT (see also fig 4). The reflex responses of the soleus muscle ( $H$ and $T)$ are well developed, while the direct motor $(M)$ response is very small. 
Table 1 Temporal features (in ms) of electrically evoked $P_{1}$ and $P_{2}$ responses at lower and middle thigh (32 subjects)

\begin{tabular}{|c|c|c|c|c|c|c|c|}
\hline \multirow[t]{2}{*}{ Recording site } & \multicolumn{4}{|c|}{ Latency at the first positive peak Latency changes } & \multicolumn{2}{|c|}{ Duration changes $\ddagger$} & \multirow{2}{*}{$\begin{array}{l}P_{1}-P_{2} \text { interwave } \\
\text { latency }\end{array}$} \\
\hline & $P_{1}$ & $P_{2}$ & $P_{1}$ & $P_{2}$ & $P_{1}$ & $P_{2}$ & \\
\hline $\begin{array}{l}\text { Lower thigh } \\
\text { Middle thigh }\end{array}$ & $\begin{array}{c}2 \cdot 10 \pm 0.38 \\
\mathrm{~A} \\
3.64 \pm 0.47 \\
\mathrm{C}\end{array}$ & $\begin{array}{c}22.05 \pm 1.76 \\
20.30 \stackrel{\mathrm{B}}{\mathrm{D}} 1.46\end{array}$ & $1.55 \pm \frac{ \pm}{E} 0.29$ & $1.75 \underset{F}{ \pm} 0.30$ & $0.36 \pm \underset{G}{ \pm} 0 \cdot 19$ & $0.13 \pm \underset{\mathrm{H}}{ \pm 0.19}$ & $\begin{array}{c}19.95 \pm 1.65 \\
16.66 \pm \frac{\mathrm{I}}{\mathrm{K}} \\
1.45\end{array}$ \\
\hline
\end{tabular}

Comparisons: A vs C: $\mathrm{t}=29.821 \dagger ; \mathrm{B}$ vs $\mathrm{D}: \mathrm{t}=32.357 \dagger ; \mathrm{E}$ vs $\mathrm{F}: \mathrm{t}=7.598 \dagger ; \mathrm{G}$ vs $\mathrm{H}: \mathrm{t}=3.519^{*} ; \mathrm{I}$ vs $\mathrm{K}: \mathrm{t}=33.795 \dagger(\dagger p<0.001$; ${ }^{*} \mathrm{p}<0.005$ )

$\ddagger$ In this and in the following table the duration of $P_{1}$ and $P_{2}$ has been determined as the time interval between the first and the last positive peak of each response

triphasic (P.N.P.) action potentials labelled $P_{1}$ and $P_{2}$ (fig $1 A$ ), the $P_{1} / P_{2}$ amplitude ratio varying from 6.6:1 at LT to $4 \cdot 4: 1$ at MT. The temporal features of $P_{1}$ and $P_{2}$ are reported in table 1 . In particular, the latency of $P_{1}$ increased in the proximal direction while $P_{2}$ showed the opposite trend. Accordingly, the time interval between $P_{1}$ and $P_{2}$ was longer at $L T$ than at MT. The increase in latency of $P_{1}$ in the proximal direction was consistently shorter than the corresponding change of $P_{2}$ in the distal direction, suggesting that $P_{1}$ and $P_{2}$ volleys are transmitted by fibres with different conduction velocities. On the contrary the increase in duration of $P_{1}$ in the proximal direction, apparently due to the temporal dispersion of the afferent volley, was longer than the corresponding change of $\mathrm{P}_{2}$ in the distal direction.

In bipolar derivations, with both recording electrodes along the nerve axis, $P_{2}$ reversed its polarity with respect to $P_{1}$ (fig $2 a$, lower trace). In double pulse experiments at an interstimulus interval of $8 \mathrm{~ms}$ there was full recovery of $P_{1}$ while, at the same interval, $\mathbf{P}_{2}$ was still absent, reappearing at an interval of $100 \mathrm{~ms}$ (fig 3).

Mechanically versus electrically evoked $P_{1}$ and $P_{2}$ volleys

In 19 out of 32 subjects a comparative evaluation of mechanically and electrically evoked neurographic responses was performed. With tendon percussion, as with electrical stimulation, there were two travelling waves the latencies of which showed the opposite trend (fig 1b), thus behaving like $P_{1}$ and $P_{2}$ respectively. $P_{1}$ was a polyphasic, low voltage potential, with duration much longer than that of $P_{2}$. The amplitude ratio $\left(P_{1} / P_{2}\right)$ was reversed with respect to electrically evoked responses, $P_{2}$ being consistently larger than $P_{1}$ both at MT and at LT (fig 1b).

A comparative evaluation of the respective temporal features (table 2) showed that latency and duration of $P_{1}$ and $P_{2}$ were significantly longer with mechanical than electrical stimulation at both LT and MT. However, the increase in latency of $P_{1}$ from LT to MT was less with tendon percussion than with

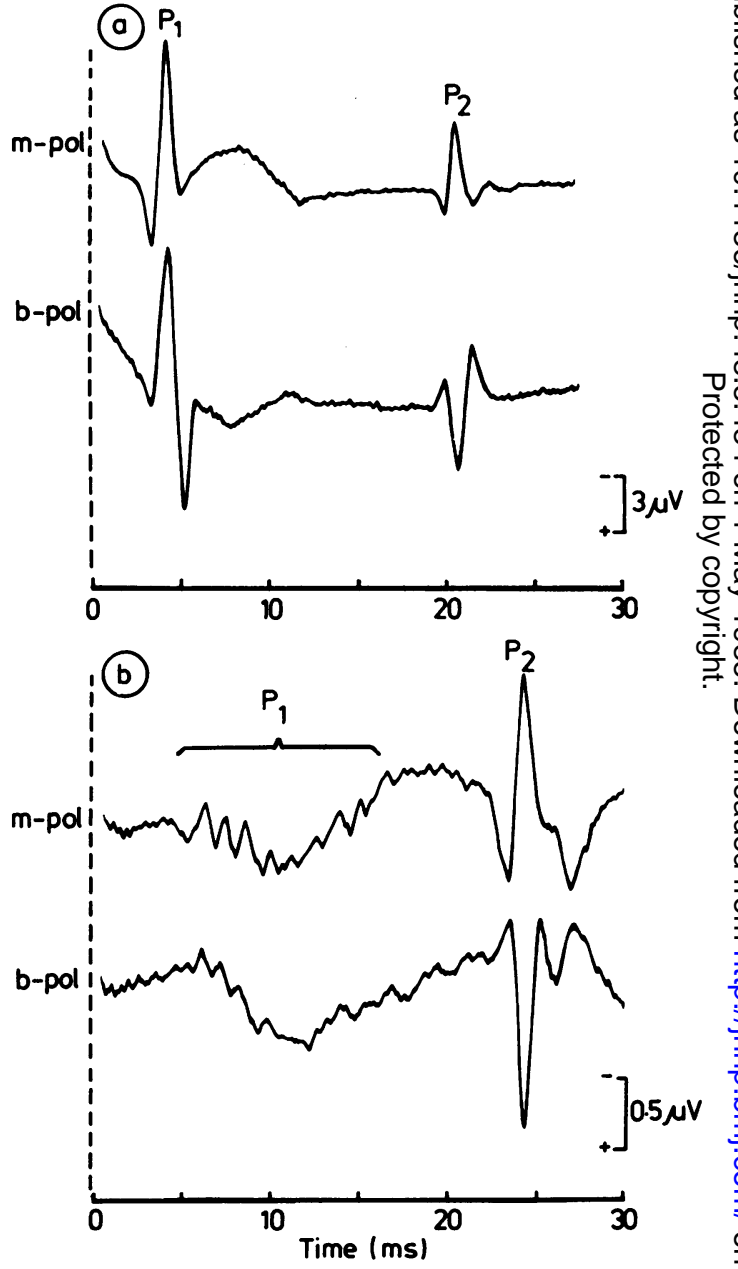

Fig 2 Electroneurographic monopolar (upper trace) and bipolar (lower trace) recordings of responses evoked (a) by electrical stimulation of the tibial nerve and (b) by percussion of the Achilles tendon. In either case bipolar recordings show a.clearcut polaritry reversal of $P_{2}$ with respect to $P_{1}$. 


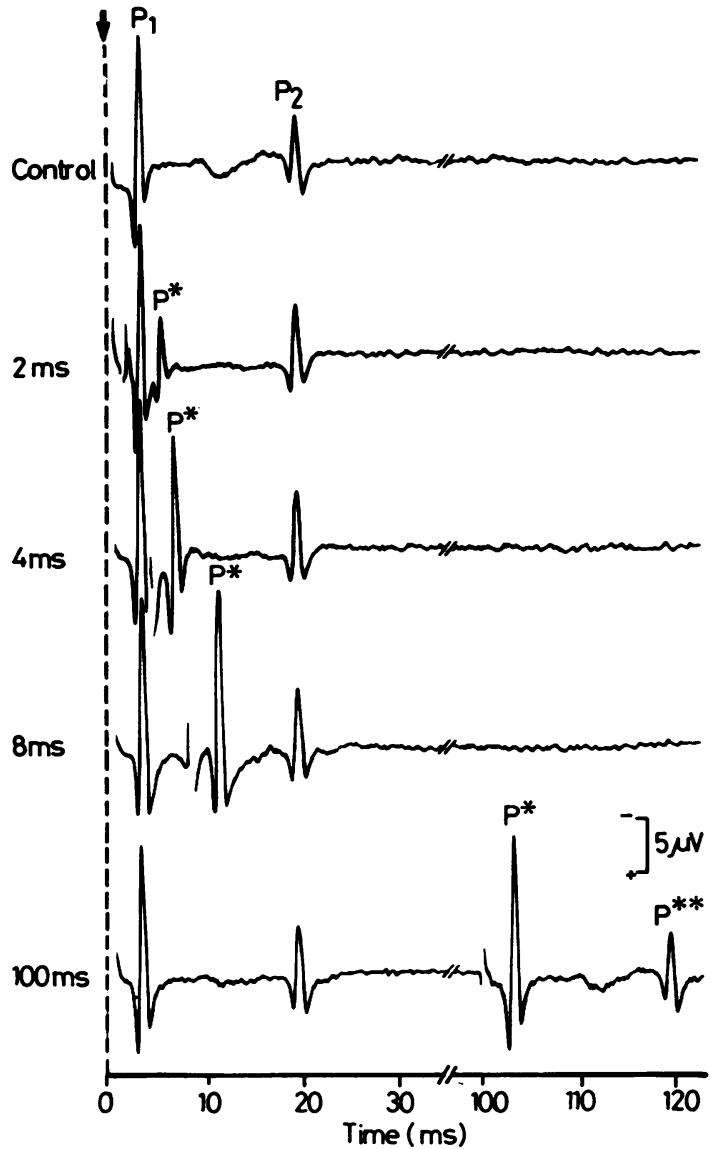

Fig 3 Double pulse experiments show that $P$, component following the second (test) stimulus $\left(P^{*}\right)$ is already visible at an interval of $4 \mathrm{~ms}$ (third trace), and completely recovered after $8 \mathrm{~ms}$ (fourth trace), while the related $P_{2}$ component $\left(P^{* *}\right)$ reappears only at an interstimulus interval of $100 \mathrm{~ms}$ (lowest trace).

electrical stimulation of the tibial nerve, suggesting some stimulus related difference in the afferent volleys. On the contrary the increase in latency of $P_{2}$ from MT to LT was apparently unrelated to the test stimulus, the same value occurring under both experimental conditions. Finally, the time interval between $P_{1}$ and $P_{2}$ was longer with tendon percussion than with electrical stimulation, both at MT and LT (fig 4). This finding could be repeated even at spinal level, the interwave latency of the lumbar response being longer on mechanical than on electrical stimulation (fig 4). When both the recording electrodes were placed along the nerve axis, $P_{2}$ reversed its polarity with respect to $P_{1}$ even when mechanical stimuli were used (fig $2 b$, lower trace).
The monosynaptic reflex and its neurographic efferent correlates under different experimental conditions

The main features of $P_{2}$ (occurrence at a stimulus intensity eliciting either the $H$ or the $T$ reflex, decrease in latency proceeding in the proximal direction irrespective of the test stimulus, opposite polarity with respect to $P_{1}$ in bipolar recordings) suggest that this potential results from activity in peripheral motor fibres discharging in the monosynaptic reflex. If this hypothesis is correct it follows that parallel changes in amplitude of the monosynaptic reflex and $P_{2}$ should occur under appropriate experimental conditions. Therefore, a battery of relevant control experiments was performed.

(1) During the $\mathrm{H}-\mathrm{H}$ conditioning experiments the $\mathrm{P}_{2}$ wave and the $\mathrm{H}$ reflex showed parallel changes, the recovery of both occurring after an interval of $100 \mathrm{~ms}$ (fig 5).

(2) When the intensity of the electrical stimulus was increased further, a progressive suppression of both $\mathrm{P}_{2}$ and the $\mathrm{H}$ reflex was observed consistently until both disappeared (fig 6a). On the contrary the amplitude of $P_{1}$ increased with stimulus intensity.

(3) A relatively slight increase in the stimulation frequency (from 0.5 to $5 \mathrm{~Hz}$ ) produced a significant reduction in amplitude of both $\mathrm{P}_{2}$ and the $\mathrm{H}$ reflex (fig 6b).

(4) During calf muscle vibration $\mathrm{P}_{2}$ and $\mathrm{H}$ reflex showed a parallel decrease in amplitude (fig 7 , lower row). The Jendrassik manoeuvre produced a clearcut facilitation of both $P_{2}$ and monosynaptic reflex (fig 7, upper and middle rows), the more striking changes being recorded with tendon jerk. It should be pointed out that neither vibration of the calf muscles nor the Jendrassik manoeuvre were able to alter the features of $P_{1}$, irrespective of the test stimulus employed.

(5) A suitably delayed stimulus $\left(\mathrm{S}_{2}\right)$ abolished the $\mathrm{P}_{2}$ component following the first stimulus $\left(\mathrm{S}_{1}\right)$ as well as the related $\mathrm{H}$ reflex, provided that $S_{2}$ was strong enough to produce the direct activation (both orthodromic and antidromic) of the efferent limb of the $\mathrm{H}$ pathway, as shown by the occurrence of a well developed $M$ response (fig $8 \mathrm{~b}$ ). When the latter was absent no visible changes of either $\mathrm{P}_{2}$ or the $\mathrm{H}$ reflex could be recorded (fig 8a, c), while proportionally intermediate variations of $\mathrm{P}_{2}$ and $\mathrm{H}$ reflex occurred when the amplitude of $M$ was lower than that of the $\mathrm{H}$ response.

All these findings point to existence of a functional relationship between $P_{2}$ and the monosynaptic reflex and confirm the hypothesis that $P_{2}$ represents the peripheral counterpart of the reflexly evoked ventral root discharge. This view was confirmed by concurrent surface and/or intrathecal recordings 
Table 2 Temporal features (in ms) of electrically ${ }^{(\mathrm{e})}$ vs mechanically ${ }^{(\mathrm{m})}$ evoked $P_{1}$ and $P_{2}$ responses recorded at lower thigh and middle thigh (19 subjects)

\begin{tabular}{|c|c|c|c|c|c|c|}
\hline \multirow[t]{2}{*}{ Recording site } & \multicolumn{4}{|c|}{ Latency at the furst positive peak } & \multicolumn{2}{|c|}{ Latency changes } \\
\hline & $\overline{P_{1} \mathrm{e}}$ & $P_{1} \mathrm{~m}$ & $P_{2} \mathrm{e}$ & $P_{2} \mathrm{~m}$ & $P_{1} \mathrm{e}$ & $P_{1} \mathrm{~m}$ \\
\hline Lower thigh & $1 \cdot 95 \pm 0 \cdot 34$ & $3 \cdot 37 \pm 0.76$ & $21 \cdot 73 \pm 1.78$ & $25 \cdot 21 \pm 2 \cdot 18$ & & \\
\hline Middle thigh & $3.54 \frac{ \pm}{B} 0.43$ & $4.82 \pm 0.83$ & $19.91 \stackrel{\frac{\mathrm{E}}{\mathrm{F}}}{\mathrm{F}} 1.64$ & $23.39 \underset{\mathrm{H}}{ \pm} 2.04$ & $1.59 \pm 0.27$ & $1.45 \underset{\mathrm{K}}{ \pm} 0.33$ \\
\hline
\end{tabular}

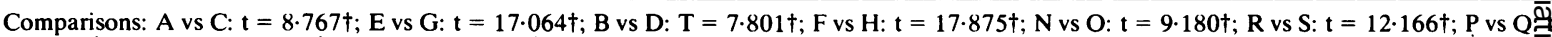
$=4.795 \dagger ; \mathrm{T}$ vs $\mathrm{U}: \mathrm{t}=6.185 \dagger ; \mathrm{I}$ vs $\mathrm{K}: \mathrm{t}=4.669 \dagger ; \mathrm{L}$ vs $\mathrm{M}: \mathrm{t}=0.006 \mathrm{~ns} ; \mathrm{X}$ vs $\mathrm{Y}: \mathrm{t}=7.516 \dagger ; \mathrm{V}$ vs $\mathrm{Z}: \mathrm{t}=7.988 \dagger ;(\dagger \mathrm{p}<0.001)$

obtained at the lumbar level under the same experimental conditions.

Speed of propagation of $P_{1}$ and $P_{2}$ volleys through the monosynaptic circuit

The speed of propagation of $P_{1}$ and $P_{2}$ volleys in the distal segment of the monosynaptic circuit was assessed by dividing the distance between LT and MT (ranging from 85 to $128 \mathrm{~mm}$ ) by the difference in latency to the first positive peak of the nerve action potential recorded at each site. Concurrent recordings of peripheral nerve and spinal root action

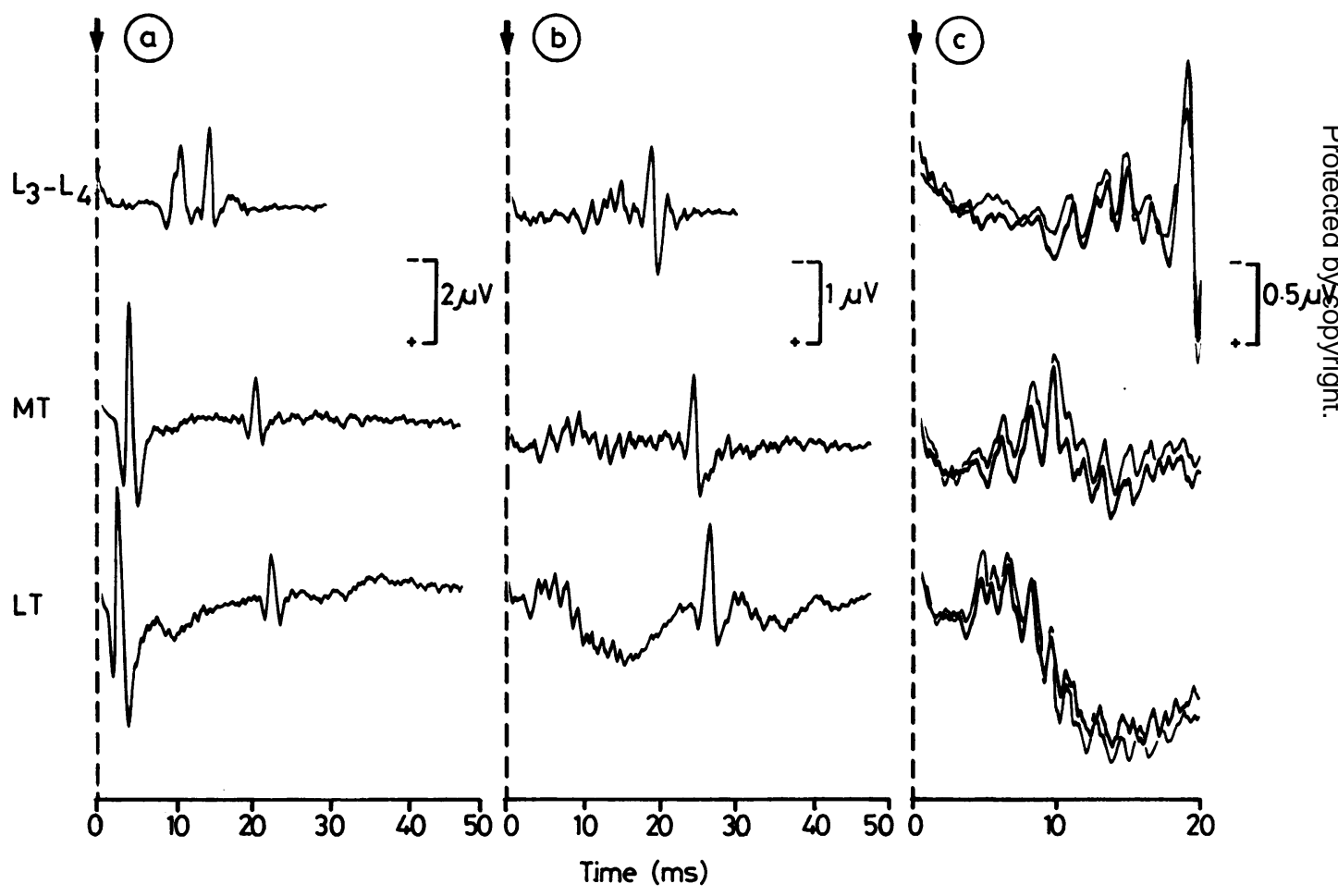

Fig 4 Concurrent monopolar recordings of spinal root (upper traces) and peripheral nerve (middle and lower traces) action potentials upon (a) stimulation of the tibial nerve and (b) percussion of the Achilles tendon. The spinal root activity has been recorded through insulated steel needles placed within the spinal theca across the interspaces L3-4.

Electroneurographic responses have been recorded from the sciatic nerve at the lower $(L T)$ and the middle (MT) thigh. It should be noted that the interpeak latency of the lumbar response as well as the $P_{1}-P_{2}$ interval at $M T$ and $L T$ are longer (b) on mechanical than (a) on electrical stimulation (see also fig 1). Two separate averages at higher display are superimposed in $c$ to show better the reproducibility of mechanically evoked $P_{1}$ component at each recording site. 


\begin{tabular}{|c|c|c|c|c|c|c|c|}
\hline \multicolumn{2}{|c|}{ Latency changes } & \multicolumn{4}{|l|}{ Duration } & \multicolumn{2}{|c|}{$P_{1}-P_{2}$ interwave latency } \\
\hline$P_{2} \bar{e}$ & $P_{2}^{\mathrm{m}}$ & $\overline{P_{1} \mathrm{e}}$ & $P_{1} \mathrm{~m}$ & $P_{2} \mathrm{e}$ & $P_{2} \mathrm{~m}$ & $P_{1}-P_{2} \mathrm{e}^{\mathrm{e}}$ & $P_{1}-P_{2} \mathrm{~m}$ \\
\hline $1.82 \pm \frac{\mathrm{L}}{0.30}$ & $1.82 \pm \underset{M}{ \pm} 0.27$ & $\begin{array}{c}1.34 \pm 0.15 \\
1.72 \pm \frac{N}{R} 0.15\end{array}$ & $\begin{array}{c}6.15 \pm 1.88 \\
7.28 \pm 1.81 \\
\mathrm{~S}\end{array}$ & $\begin{aligned} 1.85 & \pm 0.30 \\
1.72 & \frac{\mathrm{P}}{\mathrm{T}} 0.23\end{aligned}$ & $\begin{array}{l}2.68 \pm 0.72 \\
2.74 \underset{\mathrm{Q}}{ \pm} 0.67\end{array}$ & $\begin{array}{l}19.78 \pm 1.62 \\
16.36 \underset{\frac{V}{V}}{ \pm} 1.33\end{array}$ & $\begin{array}{l}21.84 \pm 2.25 \\
18.57 \frac{Y}{Z} 1.98\end{array}$ \\
\hline
\end{tabular}

potentials (fig 4) allowed direct assessment of conduction velocities in the proximal segment of the monosynaptic reflex arc by dividing the distance between MT and the spinal recording site (measured using compasses) by the difference in latency to the first positive peak of action potentials at each site. It should be pointed out that the dorsal root action potential (that is the continuation of $P_{1}$ volley at spinal level) following mechanical stimulation, could be picked up only by intrathecal recordings, owing to its very low amplitude.

Table 3 shows that (a) both on mechanical and on electrical stimulation conduction velocities were faster in the proximal than in the distal segment of the monosynaptic circuit; (b) both in the proximal and in the distal segment conduction velocity was faster in the afferent than in the efferent limb, irrespective of the stimulus used and the afferent conduction velocity was faster on mechanical than on electrical stimulation while the efferent conduction velocity was apparently independent from the test

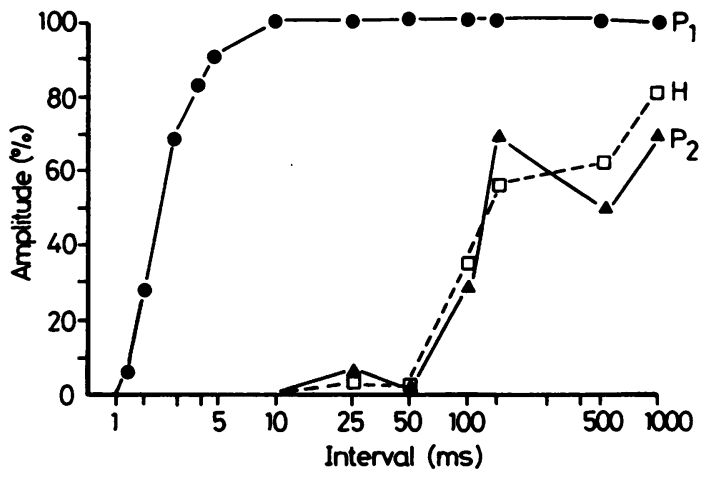

Fig 5 Relationship between response amplitude and stimulus interval showing the recovery curves of $P_{1}, P_{2}$ and $H$ reflex during an $H-H$ conditioning experiment. It should be noted that $P_{1}$ recovers much earlier than $P_{2}$ and $H$ reflex, the two latter events-showing parallel changes in amplitude. The amplitude of each response to the test stimulus is plotted as a percentage of the size of the corresponding unconditioned potential. stimulus, the respective mean values being practically identical.

The conduction velocity in the distal segment of the monosynaptic circuit, indirectly determined by changes in latency of the $\mathrm{H}$ reflex and direct motor (M) response upon stimulation of the sciatic nerve at LT and MT, was $67.96 \pm 6 \cdot 18 \mathrm{~m} / \mathrm{s}$ and $56 \cdot 38 \pm 4 \cdot 60$ $\mathrm{m} / \mathrm{s}$ respectively. Table 4 shows that the $\mathrm{H}$ conduction velocity was fairly close to the speed of propagation of electrically evoked $P_{1}$ volley, while both values were definitely lower than that extrapolated by changes in latency of mechanically evoked $P_{1}$. In contrast, a comparative evaluation of the $M$ conduction velocity with the speed of $P_{2}$ either electrically or mechanically evoked, failed to show significant differences. An indirect evaluation of the afferent and efferent conduction velocity could not be performed in the proximal segment of the monosynaptic circuit where only a mixed sensorimotor conduction index can be determined. ${ }^{8}$

\section{Discussion}

We have demonstrated that the neurographic correlates of the monosynaptic reflex are characterised by two travelling waves $\left(F_{1}\right.$ and $\left.P_{2}\right)$ whose latency increases and decreases respectively from the lowthigh to the mid-thigh sites, apparently due to the opposite direction of the underlying impulses. Both afferent $\left(P_{1}\right)$ and efferent $\left(P_{2}\right)$ responses could be obtained easily from normoreflexic subjects, provided that they were adequately relaxed, no matter whether electrical or mechanical stimuli were employed. The detection of the efferent sciatic nerve response associated with the ankle jerk and the $\mathbf{H}$ reflex did not depend on factors such as favourable experimental conditions, ${ }^{5}$ brisk tendon reflexes ${ }^{4}$ or reinforcing manoeuvres. ${ }^{7}$

When electrical stimuli are employed, the same Ia fibres are most likely activated throughout the procedure, at least in a given subject and for a given position of the stimulating electrode, and this makes the average acceptable. One may wonder whether it is appropriate to average the afferent activity following the tendon tap. In fact there could be some 

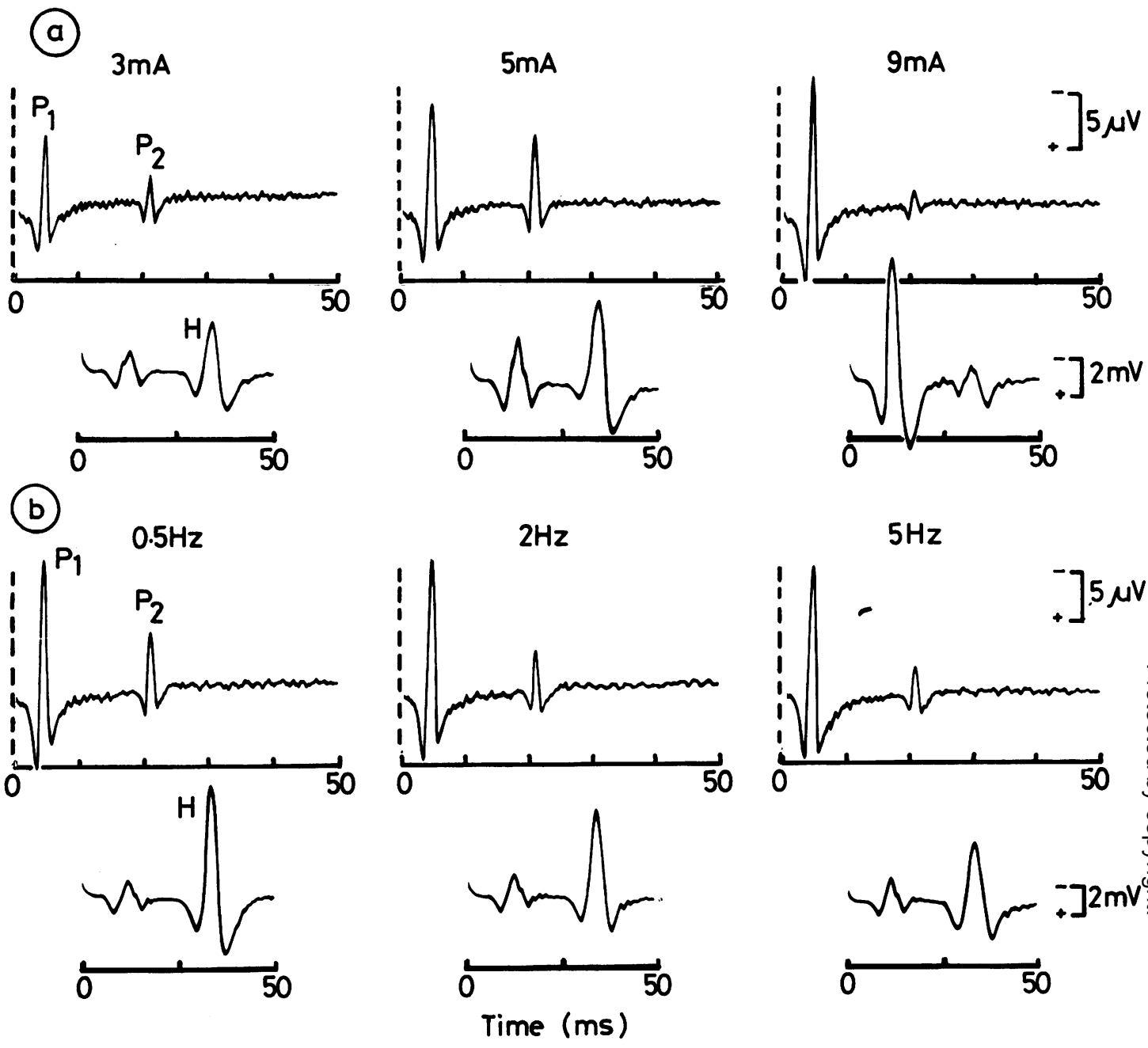

Fig $6 P_{2}$ and $H$ reflex display parallel changes in amplitude when $(A)$ the intensity or $(B)$ the frequency of the stimulus are increased.

gamma static drive, not necessarily constant during the time period needed for the averaging. Moreover both the position of the ankle and the strength of the percussion could slightly change from trial to trial. Actually the gamma static drive is not a real problem since Burke et $\mathbf{l}^{9}$ have demonstrated that there is no significant background fusimotor activity directed to non-contracting human muscles. As to the second point it must be considered that both shape and latency of the mechanically evoked $P_{1}$ were highly reproducible at each recording site (see fig 4C). Therefore we believe that the possible factors of variability of the spindle response should not invalidate our data: in particular the temporal shift of the first positive peak from LT to lumbosacral level has to be relied on.

The $P_{1}$ volley

The increase in latency of $P_{1}$ from LT to MT is the expression of an advancing wavefront of depolarisation caused by propagated action potentials in the sciatic nerve.

The polyphasic $P$, potential following the tendon tap does apparently reflect the succession of afferent discharge peaks observed both during muscle stretch $^{10} 11$ and in response to tendon percussion. ${ }^{\circ}$ 

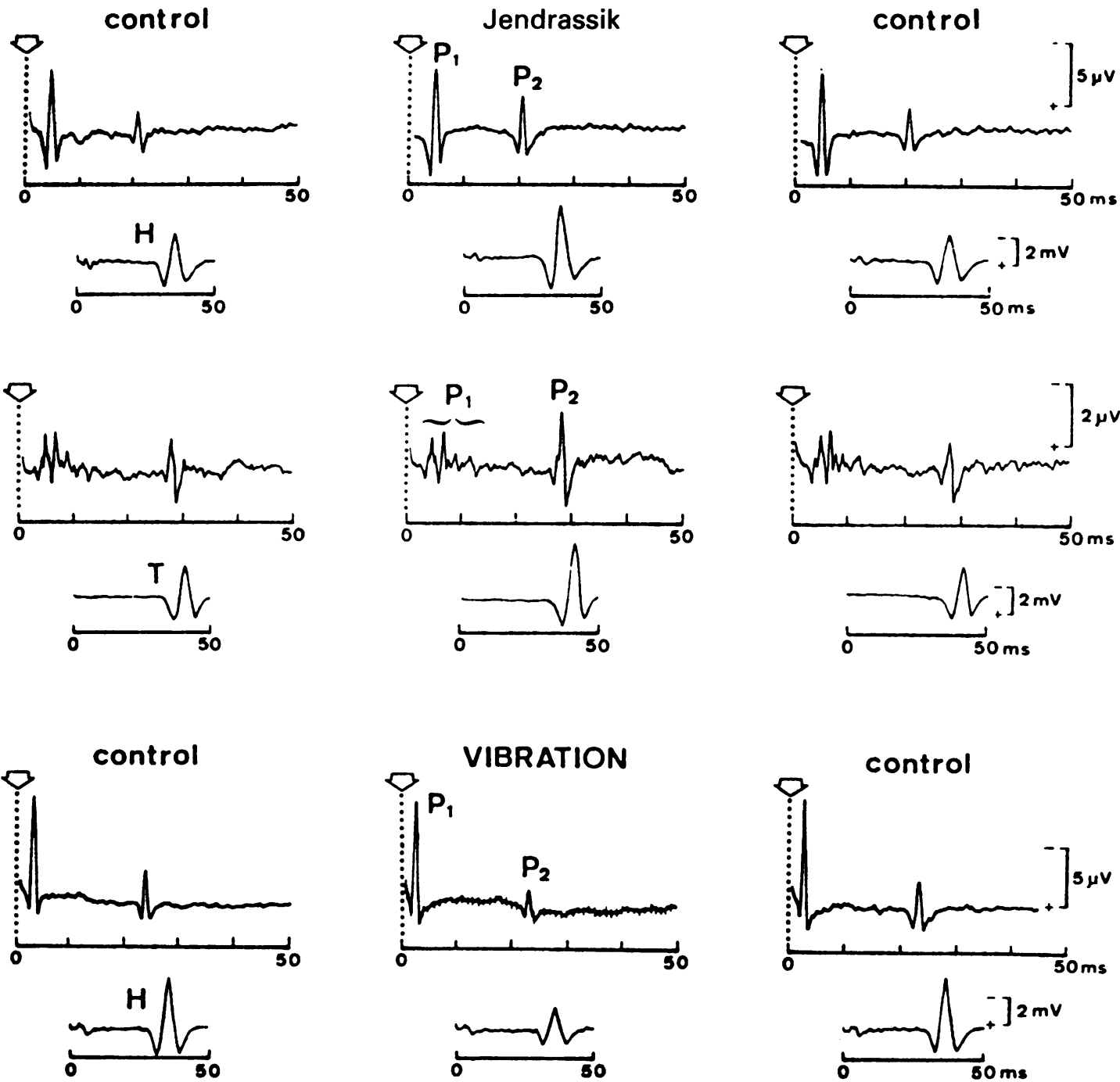

Fig 7 During the Jendrassik manoeuvre (upper and middle rows) a clearcut facilitation of $\boldsymbol{P}_{2}$ and $\mathrm{H}$ or $\mathrm{T}$ reflexes is seen, the most striking changes being recorded when the $T$ reflex is elicited; whereas during calf muscle vibration (lower row) $P_{2}$ and $H$ reflex show a parallel reduction in amplitude.

Such peaks were related either to the repetitive discharge of the same spindle endings recruited at the onset of the stretch or to successive recruitment of different spindle endings. ${ }^{10}$ In contrast the afferent volley of the $\mathrm{H}$ reflex is highly synchronous: a single centripetal impulse is evoked by the electrical shock and its neurographic correlate is a large amplitude, brief, triphasic action potential, whose first positive peak is mainly due to activation of Ia afferents.

The speed of propagation of the $P_{1}$ volley was related to the test stimulus used, being significantly faster with tendon percussion than with electrical stimulation. In fact, since the mechanically evoked $P_{1}$ is more dispersed, its first positive peak is able to reflect the small fraction of faster conducting fibres more accurately than the electrically evoked $P_{1}$. This hypothesis seems to be supported by previous studies on the distribution of conduction velocities within a nerve bundle ${ }^{12-14}$ showing that the faster contingent cannot be adequately detected on elec- 
Table 3 Speed of propagation ( $m / s)$ of $P_{1}$ and $P_{2}$ volleys through the monosynaptic reflex pathway

\begin{tabular}{|c|c|c|c|c|}
\hline \multirow[t]{3}{*}{ Stimulus } & \multicolumn{4}{|l|}{ Segments } \\
\hline & \multicolumn{2}{|l|}{ Proximal } & \multicolumn{2}{|l|}{ Distal } \\
\hline & $P_{1}$ & $P_{2}$ & $P_{1}$ & $P_{2}$ \\
\hline $\begin{array}{l}\text { Electrical } \\
\text { Mechanical }\end{array}$ & $\begin{array}{c}73.52 \pm 6.67 \\
\underset{A}{ \pm} \stackrel{ \pm}{\mathrm{C}} 9.89\end{array}$ & $\begin{array}{c}64.78 \pm 6.20 \\
\text { B } \\
64.52 \stackrel{ \pm}{\mathrm{D}} 6.14\end{array}$ & $\begin{array}{c}65.82 \pm 5.29 \\
\text { E } \\
73.02 \pm 9.33 \\
\text { G }\end{array}$ & $\begin{array}{c}57.96 \pm 4.64 \\
57.94 \underset{\mathrm{F}}{ \pm} 4.58 \\
\end{array}$ \\
\hline
\end{tabular}

Comparisons: A vs B: $\mathrm{t}=5.511 \dagger ; \mathrm{C}$ vs D: $\mathrm{t}=7.955 t ;$ A vs C: $\mathrm{t}=4.283 t ; \mathrm{B}$ vs $\mathrm{D}: \mathrm{t}=0.081 \mathrm{~ns} ; \mathrm{E}$ vs F: $\mathrm{t}=8.679 \dagger ; \mathrm{G}$ vs H: $\mathrm{t}=7.478 \dagger ; \mathrm{E}$ vs $\mathrm{G}: \mathrm{t}=4 \cdot 199 \dagger ; \mathrm{F}$ vs $\mathrm{H}: \mathrm{t}=0.015 \mathrm{~ns} ; \mathrm{C}$ vs $\mathrm{G}: \mathrm{t}=3.413^{*} ; \mathrm{D}$ vs $\mathrm{H}: \mathrm{t}=4.021 \dagger ; A$ vs $\mathrm{E}: \mathrm{t}=3.343^{*} ; \mathrm{B}$ vs $\mathrm{E}: \mathrm{t}=5.901 \dagger\left(\dagger \mathrm{p}<0.001 ;{ }^{*} \mathrm{p}<\right.$ $0 \cdot 005)$

trical stimulation, being hidden in the early phase of the compound action potential. Also the afferent conduction velocity extrapolated from the differences in latency of $\mathrm{H}$ reflexes evoked from LT and MT levels was significantly slower than that of mechanically evoked $P_{1}$ : this finding adds support to the notion that the speed of propagation of impulses in the afferent limb of the monosynaptic pathway is stimulus dependent, no matter whether the related conduction velocity has been directly or indirectly evaluated.

The discrepancy between the speed of propagation of mechanically evoked $P_{1}(73.02 \pm 9.33 \mathrm{~m} / \mathrm{s}$ from LT to MT and $80.72 \pm 9.89 \mathrm{~m} / \mathrm{s}$ from MT to the lumbosacral region) and the conduction velocity of single Ia fibres $(63.18 \pm 6.9 \mathrm{~m} / \mathrm{s})$ as determined by Mano et al, ${ }^{15}$ is only apparent. In fact the latter estimate is based on a few afferents recorded more distally, that is from the tibial nerve at the popliteal fossa, where the conduction velocity should be lower than in the sciatic nerve or in the lumbosacral plexus. To our knowledge the conduction velocities of single human afferents over the segments that we have investigated, have not yet been measured.

The long lasting duration of the mechanically evoked $P_{1}$, as well as its low amplitude, is in agreement with the high degree of asynchrony in the afferent volley due to the anatomical distribution of $\overparen{\nabla}$ the spindles (see Gassel and Diamantopoulos ${ }^{2}$ ). In fact the longitudinal spread of the muscle spindles in the triceps surae makes it unlikely that a synchron- $\vec{\circ}$

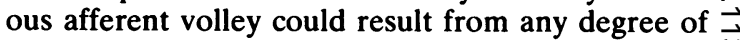
brevity of the stretch of the Achilles tendon. In addi- $\stackrel{\omega}{\sigma}$ tion the narrowing of the afferent fibre occurring near its entrance to the nuclear bag ${ }^{16}$ should be con- sidered. Differences in degree of this narrowing can + introduce another variable affecting the synchron or isation of impulses. According to Gassel and Diamantopoulos $^{2}$ even the time taken for the corr $\stackrel{\omega}{\oplus}$ duction of the wave of increased tension down th $\frac{8}{8}$ 을 muscle after the tap of the tendon might contribure to the dispersion of the afferent volley. Indeed such 3 contribution should be negligible since in non contracting muscles the propagation velocity of the. $\overrightarrow{0}$ percussion wave along the skin is rapid, exceeding of $150 \mathrm{~m} / \mathrm{s}^{6}{ }^{6}$

It could be argued that the jar produced by percussion excites sensitive mechanoreceptors in muscles throughout the limb unless widespread denervation is performed and the femur is broken, procedures which have been recommended in animal studies using comparable mechanical stimuli. ${ }^{17} \overline{\mathrm{o}}$ In man it has actually been demonstrated that a small tendon tap, subthreshold for the tendon jerk,

Table 4 Direct $\left(P_{1}\right.$ and $P_{2}$ volleys) and indirect ( $H$ and $M$ responses) assessment of afferent and efferent conduction velocity $(\mathrm{m} / \mathrm{s})$ in the distal segment of the monosynaptic reflex pathway

\begin{tabular}{|c|c|c|c|}
\hline \multirow[t]{2}{*}{ Method } & \multirow[t]{2}{*}{ Stimulus } & \multicolumn{2}{|l|}{ Conduction } \\
\hline & & Afferent & Efferent \\
\hline Direct & Electrical & $65 \cdot 82 \pm 5.29$ & $57.96 \pm \frac{B}{B} 4.64$ \\
\hline Direct & Mechanical & $73.02 \pm 9.33$ & $57.94 \underset{D}{ \pm} 4 \cdot 58$ \\
\hline Indirect & Electrical & $67.96 \pm \frac{ \pm}{E} 6 \cdot 18$ & $56.38 \pm \frac{F}{F} 4.60$ \\
\hline
\end{tabular}

Comparisons: $E$ vs $A: t=0.072, n s ; A$ vs $C: t=4 \cdot 199+; E$ vs $C: t=3.902^{*} ; F$ vs $B: t=1.496, n s ; F$ vs $D: t=1 \cdot 277$, $n s$ $(† p<0.001 * p<0.005)$ 


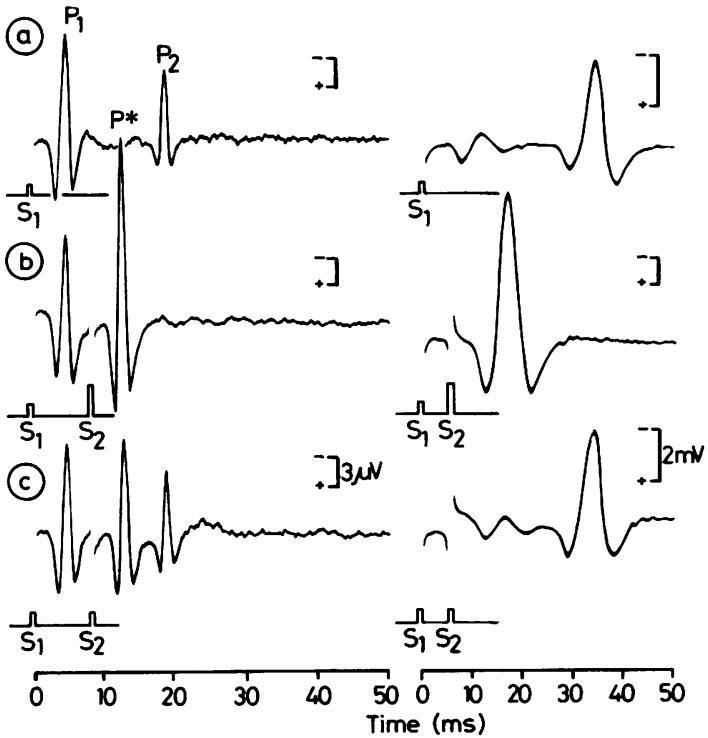

Fig 8 Electroneurographic (left row) and electromyographic (right row) responses upon $(A)$ single and $(B$ and $C$ ) double stimulation of the tibial nerve. In particular, it is shown that: $(A)$ the first stimulus $\left(S_{\mathcal{V}}\right)$ produces the usual sequence of events characterised by $P_{1}$ and $P_{2}$ volleys followed by the $H$ reflex, the $M$ response being just visible; $(B)$ a suitably delayed stimulus $\left(S_{2}\right)$ provokes the disappearance of the $P_{2}$ component following $S_{1}$ as well as of the related $H$ reflex, provided that $S_{2}$ is strong enough to evoke a well developed $M$ response; $(C)$ in the absence of the latter no visible changes of either $\mathrm{P}_{2}$ or the $\mathrm{H}$ reflex can be observed, the only visible effect of $S_{2}$ being the related $P_{1}$ component $\left(P^{*}\right)$.

diffuses from the biceps femoris to the triceps surae; ${ }^{18}$ similarly the disturbance following percussion of the Achilles tendon may well spread to the knee. However it is doubtful whether the latter manoeuvre would be an effective stimulus to spindles in thigh muscles such as biceps femoris, because the amplitude of the related percussion wave is very small when measured over the skin of the distal thigh (see fig 3 in Burke et $a l^{6}$ ). In any case the situation is quite different from that in which percussion of the biceps femoris tendon can activate triceps surae spindles ${ }^{18}$ because the latter are then very close to the percussion site (and indeed the gastrocnemii have origins on the distal femur). Summing up, we cannot exclude that spindles in biceps femoris were activated by our stimulus, but their contribution to the MT neurogram (anatomically possible since at lower thigh the nerve of the short head of the biceps has not yet joined the sciatic nerve, whereas it has done so at middle thigh) is likely to be very small, and probably not detectable with near-nerve electrodes.
The $P_{2}$ volley

The decrease in latency of $P_{2}$ from LT to MT, as well as its postsynaptic properties shown by double pulse experiments, strongly suggest that $P_{2}$ is the reflexly elicited volley in the peripheral motor fibres. Further evidence of its efferent nature was provided by the polarity reversal of $P_{2}$ with respect to $P_{1}$ when both the recording electrodes were placed along the nerve axis. In fact, when two volleys of impulses are propagating along the same nerve trunk in opposite direction, bipolar recordings will show a polarity reversal. ${ }^{3}$

Unlike $P_{1}$, the speed of propagation of $P_{2}$ failed to show stimulus-related differences: this finding suggests that the same group of fibres, namely the $\alpha$-efferent fibres, are involved in the origin of $P_{2}$ in both cases. It follows that parallel changes in amplitude of the monosynaptic reflex and $P_{2}$ should occur under appropriate experimental conditions, as indeed was the case.

When either the intensity or the frequency of the stimulus was increased, there was a significant reduction in amplitude of both $\mathrm{P}_{2}$ and the monosynaptic reflex. Calf muscle vibration decreased the amplitude of $\mathrm{P}_{2}$ and the monosynaptic reflex, while the Jendrassik manoeuvre increased both. When the efferent limb of the $\mathrm{H}$ pathway was antidromically activated by a suitably delayed stimulus of appropriate strength $\left(\mathrm{S}_{2}\right)$, both $\mathrm{P}_{2}$ and the $\mathrm{H}$ reflex following $S_{1}$ were abolished, apparently due to collision between orthodromic and antidromic impulses proximal to the recording site.

In conclusion, the constant similarity between the behaviour of $\mathrm{P}_{2}$ component and the monosynaptic reflex under different experimental conditions is consistent with the hypothesis that $P_{2}$ represents the peripheral extension of the reflexly evoked ventral root discharge. In fact, concurrent recordings of spinal root and peripheral nerve action potentials showed a satisfactory parallelism between the reflexly evoked ventral root discharge and the $P_{2}$ component.

\section{The $P_{1}-P_{2}$ interval}

The duration of $P_{2}$ following tendon tap, though significantly longer than that of electrically evoked $P_{2}$, was much shorter than the corresponding $P_{1}$ volley, thus suggesting that there was no particular dispersion of the centrifugal discharge. Indeed, Lloyd ${ }^{10}$ was able to demonstrate that a summated volley could be recorded from the ventral root on sudden mechanical stretch of the Achilles tendon in cats, although the afferent volley recorded from the dorsal root was dispersed. In his explanation he postulated that the motoneurons were facilitated by the 
early proprioceptive impulses and fired by a later component of the afferent volley, the final outcome being an increased spinal delay with respect to the $\mathrm{H}$ reflex. This hypothesis seems to agree with our observations that the time interval between $P_{1}$ and $P_{2}$ at both LT and MT levels (as well as the interpeak latency of the lumbar response) was significantly longer after the tendon tap than on electrical stimulation. In this regard it may be of relevance that the rise times of the excitatory postsynaptic potentials in soleus motoneurones following tendon percussion have been found to be longer than those resulting from electrical stimulation of the tibial nerve. ${ }^{6}$

\section{Direct and indirect assessment of conduction velocity in the monosynaptic reflex pathway}

The present study provides a more reliable method for the direct assessment of group Ia fibre conduction velocity, provided that mechanical stimuli are used. In fact, electrical stimulation of the tibial nerve leads to a consistent underestimation of the speed of propagation of impulses in the afferent limb of the monosynaptic circuit. On the contrary the efferent limb conduction velocity does not appear to be stimulus-related, the same values being obtained on either mechanical or electrical stimulation. As expected, the conduction velocity was significantly faster in the proximal than in the distal segment of the monosynaptic circuit, both in the afferent and in the efferent limb of the pathway.

An indirect evaluation of afferent and efferent conduction velocity cannot be separately obtained in the proximal segment of the monosynaptic reflex arc, where only a mixed sensorimotor conduction index could be determined. ${ }^{8}$ However, both afferent and efferent conduction velocities could be indirectly assessed in the distal segment of the monosynaptic circuit by changes in latency of $\mathrm{H}$ reflex and direct motor $(\mathrm{M})$ response upon stimulation of the sciatic nerve at LT and MT. The results thus obtained were comparable to those directly assessed by electrical stimulation of the nerve trunk, both in the afferent and the efferent limb of the monosynaptic pathway.

Summing up, neither direct nor indirect methods based on electrical stimulation of the nerve trunk are suitable to assess conduction velocity of the Ia afferents, the use of mechanical stimuli being imperative. On the contrary, both direct and indirect methods allow to determine $\alpha$-efferent conduction velocity, no matter whether electrical or mechanical stimuli are employed.

The authors are grateful to Dr David Burke for advice and comments on the manuscript.

\section{References}

${ }^{1}$ Diamantopoulos E, Gassel MM. Electrically induced monosynaptic reflexes in man. $J$ Neurol Neurosurg Psychiatry 1965;28:496-502.

2 Gassel MM, Diamantopoulos E. Mechanically and electrically elicited monosynaptic reflexes in man. $J$ Appl Physiol 1966;21:1053-8.

${ }^{3}$ Ratto S, Abbruzzese M, Abbruzzese G, Favale E. Surface-recording of the spinal ventral root discharge in man: an experimental study. Brain 1983;106:897909.

${ }^{4}$ Hagbarth K-E, Vallbo AB. Discharge characteristics of human muscle afferents during muscle stretch and contraction. Exp Neurol 1968;22:674-94.

5 Delwaide PH. Etude Expérimentelle de l'Hyperréflexie Tendineuse en Clinique Neurologique, Bruxelles, Arscia, 1971.

- Burke D, Gandevia SC, McKeon B. The afferent volleys responsible for spinal proprioceptive reflexes in man. $J$ Physiol (Lond) 1983;339:535-52.

${ }^{7}$ Burke D, McKeon B, Skuse NF. Dependence of Achilles tendon reflex on the excitability of spinal reflex pathways. Ann Neurol 1981;10:551-6.

${ }^{8}$ Troni W. Analysis of conduction velocity in the $\mathrm{H}$ pathway Part I. Methodology and results in normal subjects. J Neurol Sci 1981;51:223-33.

${ }^{9}$ Burke D, McKeon B, Skuse NF. The irrelevance of fusimotor activity to the Achilles tendon jerk of relaxed muscles. Ann Neurol 1981;10:547-50.

${ }^{10}$ Lloyd DPC. Conduction and synaptic transmission of reflex response to stretch in spinal cats. $J$ Neurophysio 1943;6:317-26.

1 Hagbarth K-E, Hagglund JV, Wallin EU, Young RR Grouped spindle and electromyographic responses t\& abrupt extension movements in man.J Physiol (Lond 1981;312:81-96.

12 Buchthal F, Rosenfalck A, Behse F. Sensory potentials of normal and diseased nerves. In: Dyck PJ, Thomas PK, Lambert EH, eds. Peripheral Neuropathies. Philadelphia, Saunders Company, 1975;442-64.

${ }^{13}$ Cummins KL, Perkel DH, Dorfman LJ. Nerve fiber conduction-velocity distributions. Estimation based on the single-fiber and compound action potentials. Electroencephalogr Clin Neurophysiol 1979;46:63446.

${ }^{14}$ Cummins KL, Dorfman LJ, Perkel DH. Nerve fiber conduction-velocity distributions. II. Estimation based on two compound action potentials. Electroencephalogr Clin Neurophysiol 1979;46:647-58.

is Mano T, Tagaki S, Mitaral G. Caractéristiques des décharges unitaires afférerents des fuseaux musculaires chez l'homme. CR Soc Biol 1976;170:500-3.

${ }^{16}$ Barker D. The innervation of the muscle spindle. $Q J$ Microscop Sci 1948;89:143-86.

${ }^{17}$ Morelli M, Nicotra L, Barnes CD, Cangiano A, Cook WA jr, Pompeiano $O$. An apparatus for producing small-amplitude high-frequency sinusoidal stretching of the muscle. Arch Ital Biol 1970;108:222-32.

${ }^{18}$ Katz R, Morin C, Pierrot-Deseilligny E, Hibino R. Conditioning of $\mathrm{H}$ reflex by a preceding subtreshold tendon reflex stimulus. $J$ Neurol Neurosurg Psychiatry 1977; 40:575-80. 